\title{
Effective dose calculation at flight altitudes with the newly computed yield function
}

\author{
Alexander Mishev*† \\ ReSolve CoE University of Oulu, Finland. \\ E-mail: alexander.misheveoulu.fi \\ Ilya Usoskin \\ ReSolve CoE University of Oulu, Finland. \\ Sodankylä Geophysical Observatory (Oulu unit), University of Oulu, Oulu, Finland. \\ E-mail: ilya.usoskin@oulu.fi
}

\begin{abstract}
An important topic in the field of space weather research is the assessment of the expected exposure of aircrew at flight altitudes due to cosmic rays (CRs), specifically during major solar energetic particle (SEPs) events. The primary cosmic ray particles induce a complicated nuclearelectromagnetic-muon cascade in the Earth atmosphere. The secondary particles form the main source of increased exposure at flight latitudes compared to the sea level. In this work we propose a numerical model for computation of the effective dose at typical commercial flight altitudes. It represents a full chain analysis, namely estimation of the solar particle spectral and angular characteristics from neutron monitor (NM) data and application of the newly computed yield function for the effective dose. The new computed yield functions for conversion of secondary particle flux to dose were obtained on the basis of extensive Monte Carlo simulation of the atmospheric cascade induced by primary protons and alpha particles and subsequent application of recently computed conversion coefficients. A comparison with the reference data is performed. A good agreement is achieved. Several example calculations are demonstrated. An application of the method for assessment of effective dose during several ground level enhancements (GLEs) is shown.
\end{abstract}

The 34th International Cosmic Ray Conference,

30 July- 6 August, 2015

The Hague, The Netherlands

\footnotetext{
* Speaker.

$\dagger$ also at Institute for Nuclear Research and Nuclear Energy, Bulgarian Academy of Sciences.
} 


\section{Introduction}

The planet Earth is constantly bombarded by high-energy particles (galactic cosmic raysGCR), mostly protons and $\alpha$-particles which penetrate deep into the atmosphere, producing large amount of secondaries. As a result aircrews are subject to increased exposure comparing to the sea level due to the increased intensity of secondary CRs. Another possible source of increasing exposure is due to sporadic source, namely by SEPs, which could produce similar atmospheric cascade, known as GLEs. At recent it was demonstrated that CRs affect the radiation environment and accordingly exposure at commercial flight altitudes, specifically during GLEs [1, 2, 月, 丹, 5]. An assessment of aircrew exposure is based on CR measurements and subsequent computations using the derived spectral and angular characteristics. Here, we describe a numerical model to estimate effective dose at flight altitudes, based on newly computed yield functions and reconstruction procedure of SEPs characteristics based on ground measurements with NMs.

\section{Model and newly computed yield functions for effective dose assessment at flight altitudes}

The dose rate can be computed as a function of the geomagnetic rigidity cut-off and altitude using a full Monte Carlo simulation of the atmospheric cascade [6]. At present, several models have been proposed, aiming to estimate the dose rate (effective and/or ambient dose equivalent) at flight altitudes due to primary CR radiation [7, 8, 9, 10, 11, 12, 13, 14,.

Because the effective dose is not a measurable quantity, International commission of radiation protection ICRP suggest the ambient dose equivalent [15] denoted as $H^{*}(d)$. It represents the dose equivalent that would be produced by the corresponding expanded and aligned field at a depth $d$ in a International Commission on Radiation Units and Measurements (ICRU) sphere (a sphere with diameter of $30 \mathrm{~cm}$ made of tissue equivalent material with a density of $1{\mathrm{~g} . \mathrm{cm}^{3}}^{3}$ and a mass composition of $76.2 \%$ Oxygen, $11.1 \%$ Carbon, $10.1 \%$ Hydrogen and $2.6 \%$ Nitrogen) on the radius vector opposing the direction of the aligned field. The unit for both effective dose and ambient dose equivalent is $\mathrm{Sv}$. The ambient dose equivalent at a depth of $\mathrm{d}=10 \mathrm{~mm} H^{*}(10)$ is recommended as a reasonable proxy for the effective dose as was shown in [16]. In fact, it slightly overestimates the effective dose, but it is not a conservative estimate for cosmic radiation exposure at aviation altitudes according to [13] and the results work presented here. Nevertheless, it is regarded as an acceptable approximation for effective dose at aircraft altitudes [17, 13].

The effective dose rate at a given atmospheric depth $h$ induced by a primary CR particle is given by:

$$
E\left(h, \lambda_{m}, \theta, \varphi\right)=\sum_{i} \int_{T^{\prime}\left(\lambda_{m}\right)}^{\infty} \int_{\Omega} J_{i}\left(T^{\prime}\right) Y_{i}\left(T^{\prime}, h\right) d \Omega d T^{\prime}
$$

where $T^{\prime}$ is the energy of the primary CR particle arriving from zenith angle $\theta$ and azimuth angle $\varphi, J_{i}\left(T^{\prime}\right)$ is the differential energy spectrum of the primary CR at the top of the atmosphere for $i$ component (proton and/or $\alpha$-particle), $\lambda_{m}$ is the geomagnetic latitude, $\Omega(\theta, \varphi)$ is a solid angle 
and $Y_{i}$ is the effective dose yield function. The corresponding effective dose yield function $Y_{i}$ is defined as

$$
Y_{i}\left(T^{\prime}, h\right)=\sum_{j} \int_{T^{*}} F_{i, j}\left(h, T^{\prime}, T^{*}, \theta, \varphi\right) C_{j}\left(T^{*}\right) d T^{*}
$$

where $C_{j}\left(T^{*}\right)$ is the fluence to effective dose conversion coefficient for a secondary particle of type $j$ (neutron, proton, $\left.\gamma, e^{-}, e^{+}, \mu^{-}, \mu^{+}, \pi^{-}, \pi^{+}\right)$with energy $T^{*}, F_{i, j}\left(h, T^{\prime}, T^{*}, \theta, \varphi\right)$ is the fluence of secondary particle of type $j$, produced by a primary particle of type $i$ (proton and/or $\alpha$-particle) with a given primary energy $T^{\prime}$. The conversion coefficients $C_{j}\left(T^{*}\right)$ for a particle of type $j$ are obtained using Monte Carlo simulations [16, 18]. The secondary particle flux produced by primary protons and $\alpha$-particles in a wide energy range is obtained on the basis of atmospheric cascade simulations using GEANT4 [19] based PLANETOCOSMICS code [20]. The yield function considers the complexity of the atmospheric cascade development, since it brings information of particle fluence and spectrum at a given altitude in the atmosphere, considering the secondary particle attenuation. The newly computed effective dose yield function for $35 \mathrm{kft}$ (typical commercial flight altitude $\approx 10.5 \mathrm{~km}$ ) is shown in Fig. 1 .

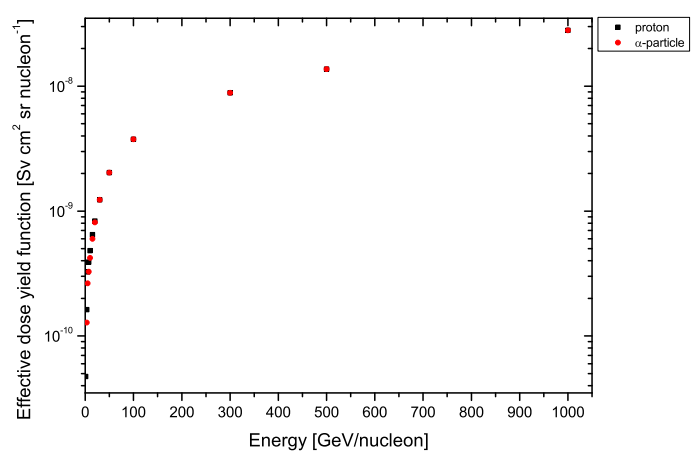

(a) Effective dose yield function for protons and $\alpha$-particles

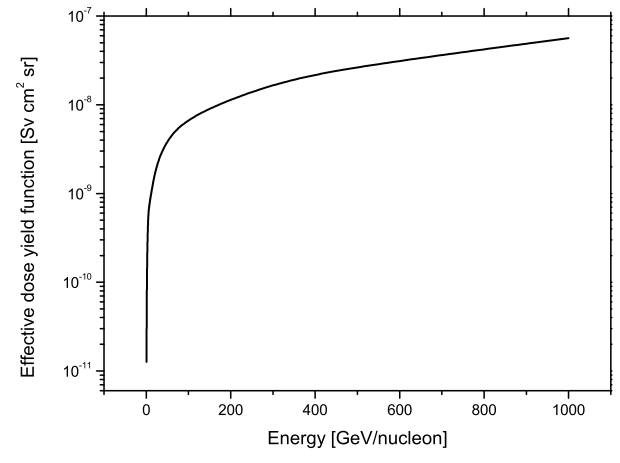

(b) Total Effective dose yield function

Figure 1: Effective dose yield function as a function of the energy per nucleon for primary CR protons and $\alpha$-particles at $35 \mathrm{kft}$ (altitude of $\approx 10.5 \mathrm{~km}$ ).

Details concerning the computation including look-up tables are given elsewhere [21].

\section{Application of the model}

Since the ambient dose equivalent is acceptable approximation for effective dose at aircraft altitudes, we perform a comparison of our model with reference data. The computed effective dose $E$ compared with reference data [22] at the altitude of $35 \mathrm{kft}$ a.s.l. for several periods is shown in Fig. 2. Here (Eq. 2.1) we assume the force field model for GCR with the corresponding modulation parameter [23]. We achieve a good agreement between the model computations and the reference data. 


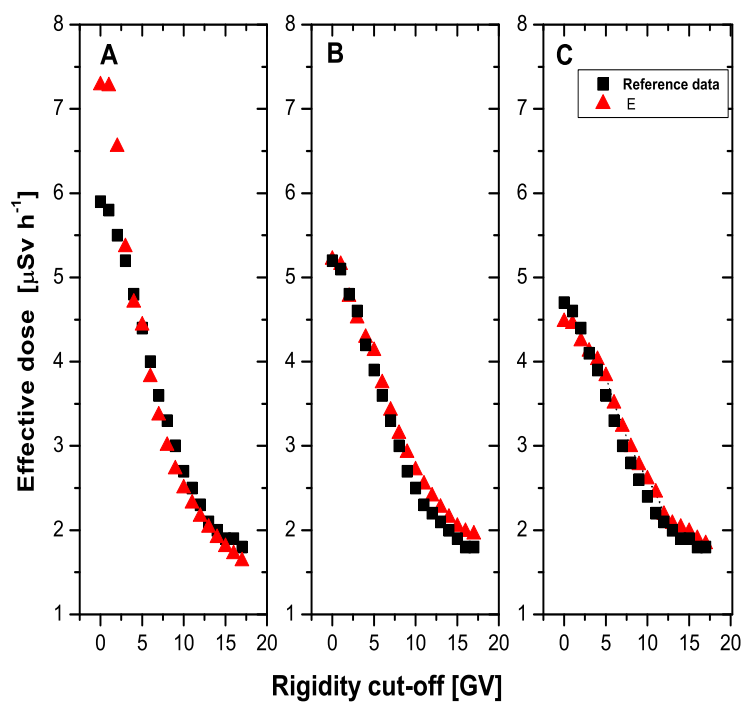

Figure 2: Computed effective dose $E$ and reference data [22] at the altitude of $35 \mathrm{kft}$ a.s.l. a) January 1998 b) January 2000 c) January 2002.

During major GLEs, the exposure is a superposition of the contribution of GCRs and SEPs, which possess an essential anisotropic part during the event onset. Therefore, as the first step the spectra SEPs should be determined outside the magnetosphere. In order to consider explicitly the anisotropy we compute the asymptotic cones in the region of interest in a grid of $5^{\circ} \times 5^{\circ}$ with the MAGNETOCOSMICS [20].

Here we apply derived spectral and angular characteristics of GLE particles obtained on the basis of NM data [24] using newly computed NM yield function [25]. Details are given elsewhere in this volume. The effective dose rate during various periods of GLE 70 is shown in Fig.3, accordingly for GLE 59 in Fig.4. During the initial phase of GLE 70 the computed effective dose was about 40-50 $\mu \mathrm{Sv} \cdot \mathrm{h}^{-1}$, while during the main and late phases the contribution of SEPs to the dose is comparable to the average due to GCR. The computed effective dose during the initial phase of GLE 59 was about $30 \mu \mathrm{Sv}^{-1}{ }^{-1}$. It diminished to about $14 \mu \mathrm{Sv}^{-\mathrm{h}^{-1}}$ during the main and late phase of the event.

\section{Conclusion}

Here we present a new full chain analysis model for assessment of effective dose at flight altitudes. The model is based on NM data and newly computed yield functions. The model demonstrates good agreement with reference data for GCRs and it is sucesfully applied for computation of effective dose rate during two GLEs. The model is fully operational and could be used for space weather applications. 

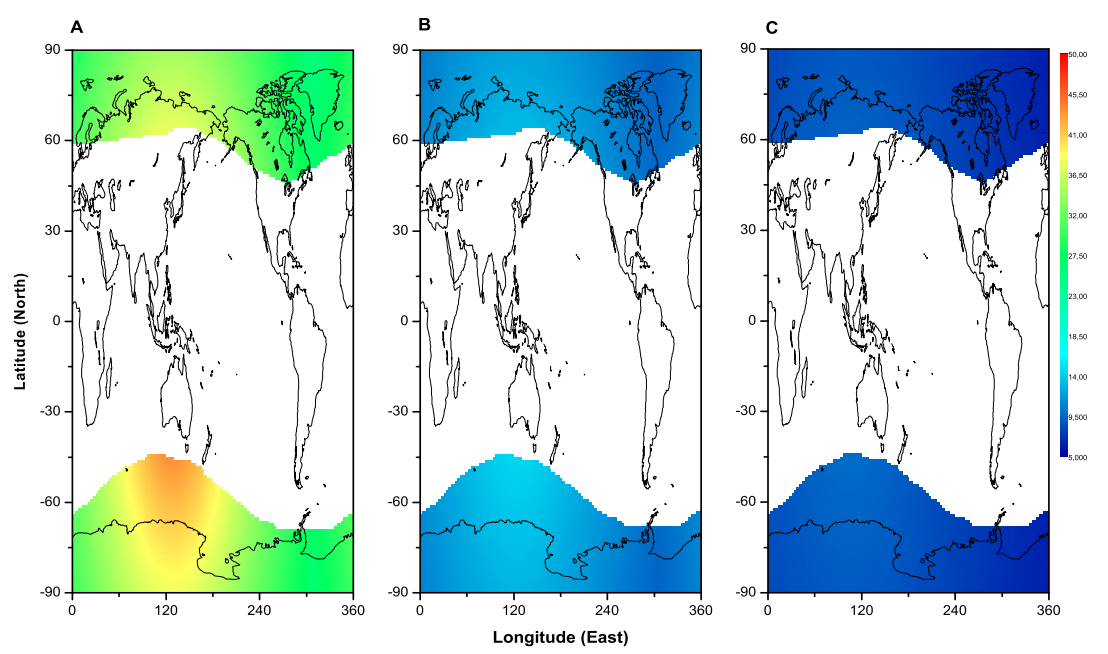

Figure 3: The effective dose rate $\left[\mu \mathrm{Sv} \mathrm{h}^{-1}\right]$ at the altitude of $35 \mathrm{kft}$ a.s.l. during the GLE 70 on 13 December 2006 in a region with $R_{c} \leq 1 \mathrm{GV}$. a) initial phase of the event; b) main phase of the event; c) late phase of the event.

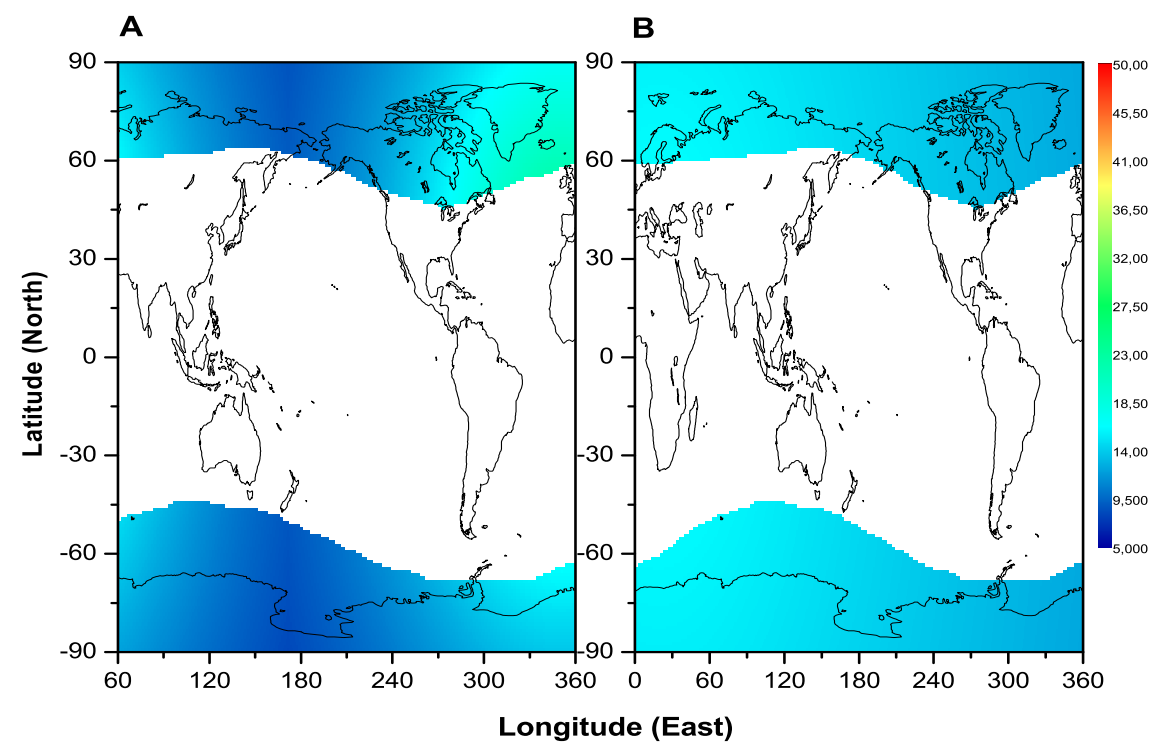

Figure 4: The effective dose rate $\left[\mu \mathrm{Sv} \mathrm{h}^{-1}\right]$ at the altitude of $35 \mathrm{kft}$ a.s.l. during the GLE 59 on 14 July 2000 in the region with $R_{c} \leq 1 \mathrm{GV}$. a) event onset; b) main phase of the event. 


\section{Acknowledgements}

We acknowledge the financial support by the Academy of Finland to the ReSoLVE Center of Excellence (project No. 272157).

\section{References}

[1] K. O'Brien, W. Friedberg, H. Sauer, and D. Smart, Atmospheric cosmic rays and solar energetic particles at aircraft altitudes, Environment International 22 (1997), no. SUPPL. 1 S9-S44.

[2] M. Shea and D. Smart, Cosmic ray implications for human health, Space Science Reviews 93 (2000), no. 1-2 187-205.

[3] F. Spurny, T.Dachev, and K. Kudela, Increase of onboard aircraft exposure level during a solar flare, Nuclear Energy Safety 10 (2002), no. 48 396-400.

[4] M. Shea and D. Smart, Space weather and the ground-level solar proton events of the 23rd solar cycle, Space Science Reviews 171 (2012) 161-188.

[5] A. Mishev, F. Adibpour, I. Usoskin, and E. Felsberger, Computation of dose rate at flight altitudes during ground level enhancements no. 69, 70 and 71, Advances in Space Research 55 (2015), no. 1 354-362.

[6] A. Ferrari, M. Pelliccioni, and T. Rancati, Calculation of the radiation environment caused by galactic cosmic rays for determining air crew exposure, Radiation Protection Dosimetry 93 (2001), no. 2 $101-114$.

[7] H. Schraube, G. Leuthold, W. Heinrich, S. Roesler, and D. Combecher, European program package for the calculation of aviation route doses, version 3.0, Tech. Rep. D-85758, National Research Center for Environment and Health Institute of Radiation Protection, Neuherberg, Germany, 2000.

[8] S. Roesler, W. Heinrich, and H. Schraube, Monte carlo calculation of the radiation field at aircraft altitudes, Radiation Protection Dosimetry 98 (2002), no. 4 367-388.

[9] B. Lewis, L. Bennett, A. Green, A. Butler, M. Desormeaux, F. Kitching, M. McCall, B. Ellaschuk, and M. Pierre, Aircrew dosimetry using the predictive code for aircrew radiation exposure (pcaire), Radiation Protection Dosimetry 116 (2005), no. 1-4 320-326.

[10] T. Sato, H. Yasuda, K. Niita, A. Endo, and L. Sihver, Development of parma: Phits-based analytical radiation model in the atmosphere, Radiation Research 170 (2008) 244-259.

[11] D. Matthiä, L. Sihver, and M. Meier, Monte-carlo calculations of particle fluences and neutron effective dose rates in the atmosphere, Radiation Protection Dosimetry 131 (2008), no. 222-228.

[12] A. Mishev and E. Hristova, Recent gamma background measurements at high mountain altitude, Journal of Environmental Radioactivity 113 (2012) 77-82.

[13] C. Mertens, M. Meier, S. Brown, R. Norman, and X. Xu, Nairas aircraft radiation model development, dose climatology, and initial validation, Space Weather 11 (2013), no. 10 603-635.

[14] A. Mishev, Computation of radiation environment during ground level enhancements 65, 69 and 70 at equatorial region and fight altitudes, Advances in Space Research 54 (2014), no. 3 528-535.

[15] ICRP, ICRP publication 103: The 2007 recommendations of the international commission on radiological protection., Annals of the ICRP 37 (2007), no. 2-4. 
[16] M. Pelliccioni, Overview of fluence-to-effective dose and fluence-to-ambient dose equivalent conversion coefficients for high energy radiation calculated using the fluka code, Radiation Protection Dosimetry 88 (2000), no. 4 279-297.

[17] M. Meier, M. Hubiak, D. Matthiä, M. Wirtz, and G. Reitz, Dosimetry at aviation altitudes (2006-2008), Radiation Protection Dosimetry 136 (2009), no. 4 1-35.

[18] N. Petoussi-Henss, W. Bolch, K. Eckerman, A. Endo, N. Hertel, J. Hunt, M. Pelliccioni, H. Schlattl, and M. Zankl, Conversion coefficients for radiological protection quantities for external radiation exposures, Annals of the ICRP 40 (2010), no. 2-5 1-257.

[19] S. Agostinelli, J. Allison, and K. Amako, Geant4 - a simulation toolkit, Nuclear Instruments and Methods in Physics Research Section A: Accelerators, Spectrometers, Detectors and Associated Equipment 506 (2003), no. 3 250-303.

[20] L. Desorgher, E. Flückiger, M. Gurtner, M. Moser, and R. Bütikofer, A geant 4 code for computing the interaction of cosmic rays with the earth's atmosphere, Internationl Journal of Modern Physics A 20 (2005), no. A11 6802-6804.

[21] A. Mishev and I. Usoskin, Numerical model for computation of effective and ambient dose equivalent at flight altitudes. application for dose assessment during gles, Journal of Space Weather and Space Climate 5 (2015) A10.

[22] H. Menzel, The international commission on radiation units and measurements, Journal of the ICRU 10 (2010), no. 2 1-35.

[23] I. Usoskin, G. Bazilevskaya, and G. A. Kovaltsov, Solar modulation parameter for cosmic rays since 1936 reconstructed from ground-based neutron monitors and ionization chambers, Journal of Geophysical Research 116 (2011) A02104.

[24] A. Mishev, L. Kocharov, and I. Usoskin, Analysis of the ground level enhancement on 17 may 2012 using data from the global neutron monitor network, Journal of Geophysical Research 119 (2014) 670-679.

[25] A. Mishev, I. Usoskin, and G. Kovaltsov, Neutron monitor yield function: New improved computations, Journal of Geophysical Research 118 (2013) 2783-2788. 